\title{
Man-made synthesis of ultrafine photoluminescent nanodiamonds containing less than three silicon-vacancy color centers
}

ChengKe Chen ${ }^{a}$, YingShuang Mei a , JinMing Cui b,c, Xiao Li ${ }^{\text {a }}$, MeiYan Jiang ${ }^{\text {a }}$, ShaoHua $\mathrm{Lu}^{\text {a }}$, XiaoJun $\mathrm{Hu}^{\mathrm{a}, *}$

${ }^{a}$ College of Materials Science and Engineering, Zhejiang University of Technology, Hangzhou, Zhejiang Province, 310014, China

b CAS Key Laboratory of Quantum Information, University of Science and Technology of China, Hefei 230026, China

c Synergetic Innovation Center of Quantum Information and Quantum Physics, University of Science and Technology of China, Hefei 230026, China

ABSTRACT: Silicon-vacancy (SiV) centred diamond exhibits near infrared emission at $738 \mathrm{~nm}$ with narrower zero-phonon line and shorter lifetime comparative with nitrogen-vacancy (NV) center, considered as a promising room-temperature single-photon source. However, unlike NV centred nanodiamonds easily obtained by detonation, man-made synthesis of ultrafine $\mathrm{SiV}$ nanodiamonds is a key challenge. Here, we provide a controllably method for synthesizing nanodiamonds with one or few $\mathrm{SiV}$ emitters by reducing their size to the near minimum via oxygen plasma treatments. The size of nanodiamond is uniformly reduced from 11 to $1.7 \mathrm{~nm}$ under

* Corresponding author. Tel: +86 571-88871522, E-mail: huxj@zjut.edu.cn (Xiaojun Hu) 
different treatment durations. It is found that nanodiamonds of a size between 2.1 and $4 \mathrm{~nm}$ contain fewer than three emitters. Size dependence of microstructure and photoluminescence suggests that $\mathrm{SiV}$ centers exist in the outer shell of nanodiamonds with diameter larger than $\sim 2 \mathrm{~nm}$. This material has significant applications in nanophotonics platform, quantum communications and quantum photonics.

\section{Introduction}

Fluorescent centres in diamond have significant applications in fields such as quantum communications, quantum photonics, and biological imaging. Fluorescent diamond is generally considered as a photostable solid-state single photons source at room temperature, supplying prominent candidates for generating and manipulating quantum states of light. In addition, the stability of the diamond defects constitutes the main advantage over other systems [1]. Furthermore, nanodiamonds are biocompatible, optically stable, anticorrosion and easy to surface functionalize, making them ideally suited for biological applications, catalyst and catalyst carrier [2-4].

Out of more than 500 colour centres, fewer than ten centres in diamond have thus far been identified as bright, stable single-photon emitters [1]. Of these centres, nitrogen-vacancy (NV) centres have been thoroughly studied [5-11]. Electrically neutral $\left(\mathrm{NV}^{0}\right)$ and negatively charged $\left(\mathrm{NV}^{-}\right)$centers exhibit one zero-phonon line (ZPL) at $575 \mathrm{~nm}$ and $637 \mathrm{~nm}$ with a characteristic broad phonon sideband that extends from 580 to $650 \mathrm{~nm}$ and to $800 \mathrm{~nm}$, respectively. The wide emission band and long lifetime of NV centres, up to $25 \mathrm{~ns}$, renders them unsuitable for single-photon-source 
[12-14]. In contrast, the silicon-vacancy ( $\mathrm{SiV})$ centre has a ZPL centred at $738 \mathrm{~nm}$ $(1.681 \mathrm{eV})$, with a very narrow band $(\sim 5 \mathrm{~nm})$ and short luminescence lifetime $(\sim 1.2 \mathrm{~ns})$ $[1,15,16]$. These characteristics facilitate the excellent performance of $\mathrm{SiV}$ centered diamond, which have recently attracted increasing attentions.

It is possible to obtain one $\mathrm{SiV}$ centre in one diamond grain small enough in size to support a single-photon source. The calculation based on the first-principles density functional theory showed that isolated diamond nanoparticles as small as $1.6 \mathrm{~nm}$, were capable of housing stable photoluminescent $\mathrm{SiV}$ centres. It was also found that molecular-sized $(\sim 1.6 \mathrm{~nm})$ fluorescent $\mathrm{SiV}$ nanodiamonds containing approximately 3 emitters existed in meteorites [17]. However, unlike NV centred nanodiamonds easily obtained by detonation [8, 9], the man-made synthesis of SiV centred ultrafine nanodiamonds is so far an outstanding challenge. This dramatically limits the related research and applications.

Here, we provide a controllably method for preparing ultrafine nanodiamonds with one or few $\mathrm{SiV}$ emitters by reducing the diamond grain size in nanocrystalline diamond films to the near minimum via oxygen plasma treatments. Then, the photon statistics of $\mathrm{SiV}$ fluorescence in these man-made nanodiamond grains were investigated. The results show that the size of diamond grains uniformly decreases from 11 to $1.7 \mathrm{~nm}$ via different treatment durations. The finest diamond grains that can emit $\mathrm{SiV}$ PL are in the size range of 2.1-4 nm, and these grains were separated from the film and immobilized on a fused silica slide. Hanbury Brown-Twiss (HBT) measurements show that fewer than three emitters are present within the fluorescent 
spot, indicating that sub-4-nm nanodiamond grains with few SiV emitters are prepared. This method is of significant importance for the applications of $\mathrm{SiV}$ photoluminescent diamonds as nanophotonics platform, quantum communications and quantum photonics.

\section{Results and discussion}

Figure 1 (a) shows photoluminescent spectra in the range of $720-760 \mathrm{~nm}$ of the nanocrystalline diamond films treated with oxygen plasma for different durations, denoted $\mathrm{O}-\mathrm{n}$, where $\mathrm{n}$ is the treatment duration. The untreated nanocrystalline diamond film (black line, nearly the same with the blue line and magenta line) shows a $\mathrm{SiV}$ peak meaning that the diamond grains contain $\mathrm{SiV}$ centers, because silicon atoms diffuse from the silicon substrate into the growing diamond crystals $[18,19]$. After a 5-min oxygen plasma treatment, the intensity of the luminescent $\mathrm{SiV}$ peak is enhanced, as shown in Figure 1 (a), and the red peak is obviously higher than the others. As the treatment duration increases from 10 to $20 \mathrm{~min}$, the luminescent $\mathrm{SiV}$ peak weakens. After 30 min treatment, the peak disappears.
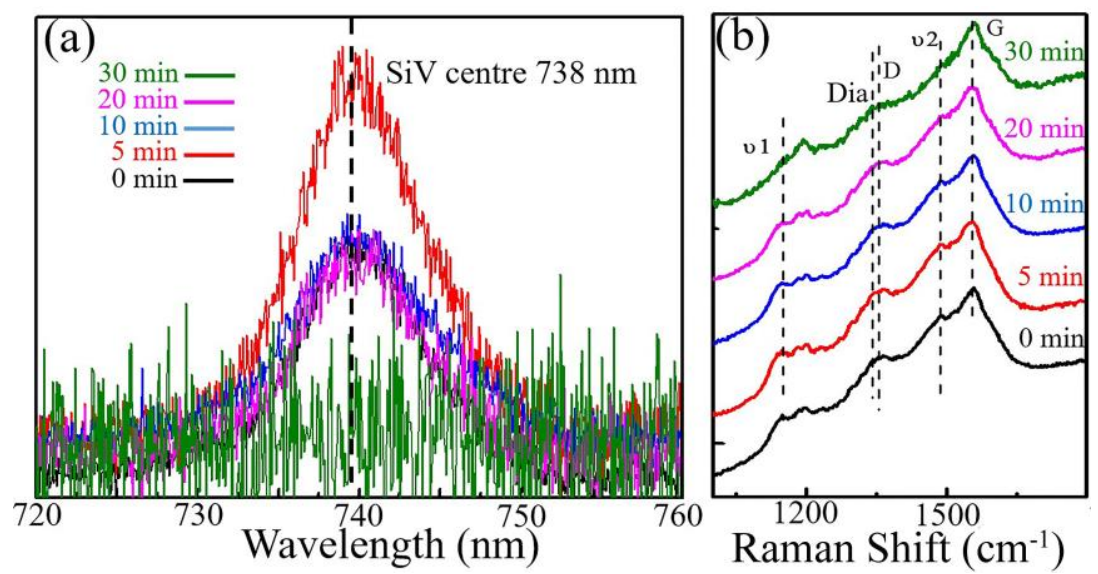

Figure 1. (a) Photoluminescence (PL) and (b) Raman spectra of the nanocrystalline diamond films treated with oxygen plasma for 0 to $30 \mathrm{~min}$. 
Figure 1 (b) shows the Raman spectra in the range of $1000-1800 \mathrm{~cm}^{-1}$ of the nanocrystalline diamond films treated with oxygen plasma for different durations. Each Raman spectrum in Figure 1 (b) shows the typical characteristics of nanocrystalline diamond films with a grain size smaller than $10 \mathrm{~nm}$, and five obvious peaks were labelled by vertical dashed lines. The peaks at approximately $1140(v 1)$ and $1480(\mathrm{v} 2) \mathrm{cm}^{-1}$ are related to trans-polyacetylene (TPA) and can be assigned to the $\mathrm{C}-\mathrm{H}$ bending and/or $\mathrm{C}-\mathrm{C}$ stretching modes and the $\mathrm{C}=\mathrm{C}$ stretching mode of the TPA chains, respectively $[20,21]$. The peak at $1332 \mathrm{~cm}^{-1}$, known as the diamond peak, is very weak, indicating small size of diamond grains. In addition, $\mathrm{sp}^{2}$ graphitic phases are visible at 1340 (D band) and $1560 \mathrm{~cm}^{-1}$ (G band) [21, 22].

The Raman spectra of the O-0, O-5, O-10 and O-20 samples show very few differences, indicating that their phase composition continuously evolves. After 30 min of oxygen plasma etching, the diamond peak is nearly undetectable, indicating a further decrease in the content or grain size of the diamond. This leads to the disappearance of the SiV PL in the O-30 sample. As oxygen plasma can oxidize the film, different treatment durations will alter the film compositions and structures, thereby affecting the SiV PL. To analyse the effects of oxygen plasma treatment on the composition of the film, we collected the X-ray photoelectron spectroscopy (XPS) wide scan spectra and the $\mathrm{C} 1$ s core-energy level spectra of the O-0, O-5, O-20 and O-30 samples, as shown in Figure 2. 


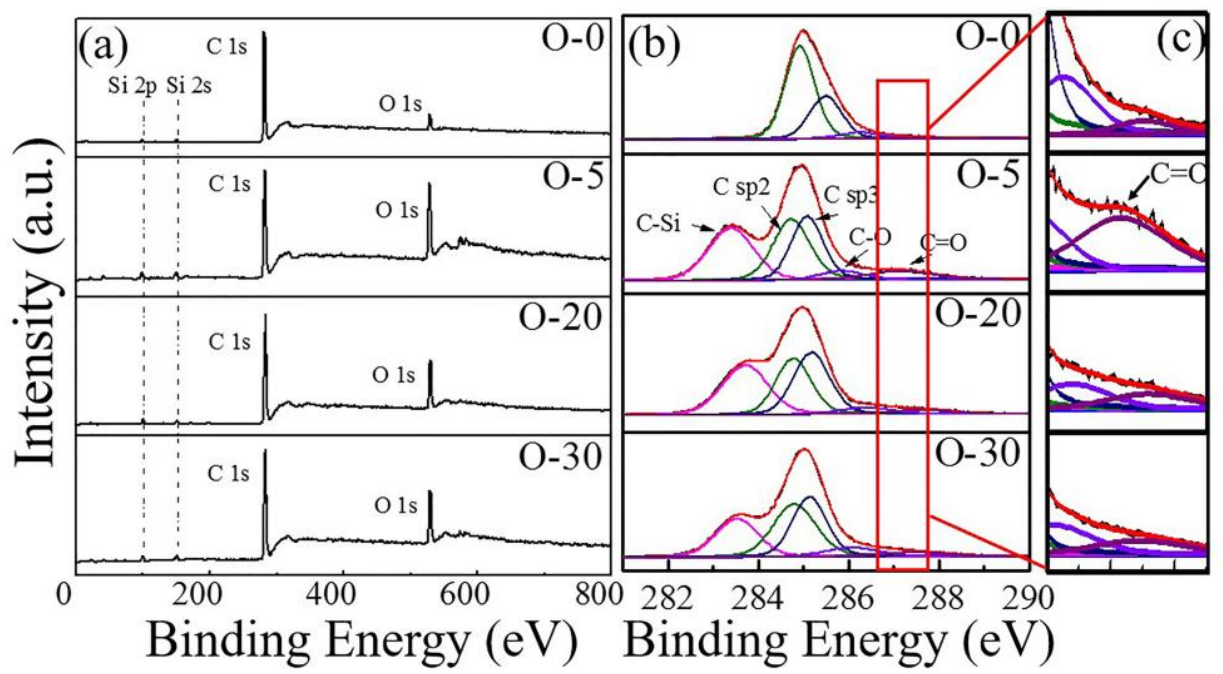

Figure 2. (a) X-ray photoelectron spectroscopy (XPS) wide scan spectra and (b) C 1s core-energy level spectra and their deconvolution of the O-0, O-5, O-20 and O-30 samples. (c) is the enlarged images of the red square in (b).

Figure 2 (a) shows that the $\mathrm{O} 1 \mathrm{~s}$ peak of the $\mathrm{O}-5$ sample is much stronger than that of the O-0 sample and slightly stronger than those of the other samples. This indicates that more oxygen-related bonds are present on the surface of the nanocrystalline diamond films after treatment and that the O-5 sample has the highest content of oxygen-related bonds. We further analysed the C 1s core-energy level spectra, which were deconvoluted into five components at 283.5, 284.8, 285.2, 286.3 and $287.0 \mathrm{eV}$, attributed to $\mathrm{C}-\mathrm{Si}, \mathrm{sp}^{2}$ carbon $(\mathrm{C}=\mathrm{C}), \mathrm{sp}^{3}$ bulk carbon $(\mathrm{C}-\mathrm{C}), \mathrm{C}-\mathrm{O}$ and $\mathrm{C}=\mathrm{O}$ bonds, respectively [23-25]. The oxygen plasma-treated samples exhibit a C-Si bond peak, which may originate from the exposed silicon substrate owing to the oxygen plasma treatment. In addition, we enlarged the spectral range from $286.0-288.5 \mathrm{eV}$, related to $\mathrm{C}=\mathrm{O}$ bonds, as shown in Figure 2(c). The $\mathrm{C}=\mathrm{O}$ bond peak is much stronger for the O-5 sample than for the O-0, O-20 and O-30 samples. This result is consistent with the changes in the $\mathrm{SiV}$ PL of the samples, suggesting that $\mathrm{C}=\mathrm{O}$ bonds tend to increase 
the intensity of the SiV PL. This finding agrees with those of our previous work [26], in which $\mathrm{C}=\mathrm{O}$ bonds produced a much larger positive electron affinity, trapping the excited-state electrons and allowing them to scatter back to the ground state, resulting in $\operatorname{SiV}$ PL $[27,28]$.

Comparing the XPS results of the O-20 and O-30 samples, we find that their surface composition is nearly the same. However, the O-20 sample exhibits SiV PL, while the O-30 sample does not, indicating that in this case, the surface termination is not the decisive factor for the disappearance of $\mathrm{SiV}$ PL in the O-30 sample. To further understand the correlation between oxygen plasma etching and SiV PL, we analysed the microstructural evolution of the samples under different conditions using high-resolution transmission electron microscopy (HRTEM). Figure 3 shows low-magnification HRTEM images of the samples O-5, O-10, O-20 and O-30, which illustrate that nanocrystalline diamond grains are uniformly distributed in the film. And the particle size distribution of the samples O-5, O-10, O-20 and O-30 were shown on the right of Figure 3. The grain size is approximately 7-11 nm, 3.5-6.2 nm, 2.1-4 $\mathrm{nm}$ and 1.7-2.3 $\mathrm{nm}$ for the O-5, O-10, O-20 and O-30 samples, respectively, indicating that the diamond grain size decreases with increasing oxygen plasma treatment duration. This relation leads to a dramatic decrease in the diamond content in the O-30 sample, which is in good agreement with the disappearance of the diamond peak in its Raman spectrum. More importantly, comparing the results of the grain size and PL spectra, the finest grains that can emit SiV PL are 2.1-4 nm in size. These are currently the smallest man-made nanodiamonds that can emit SiV PL. 

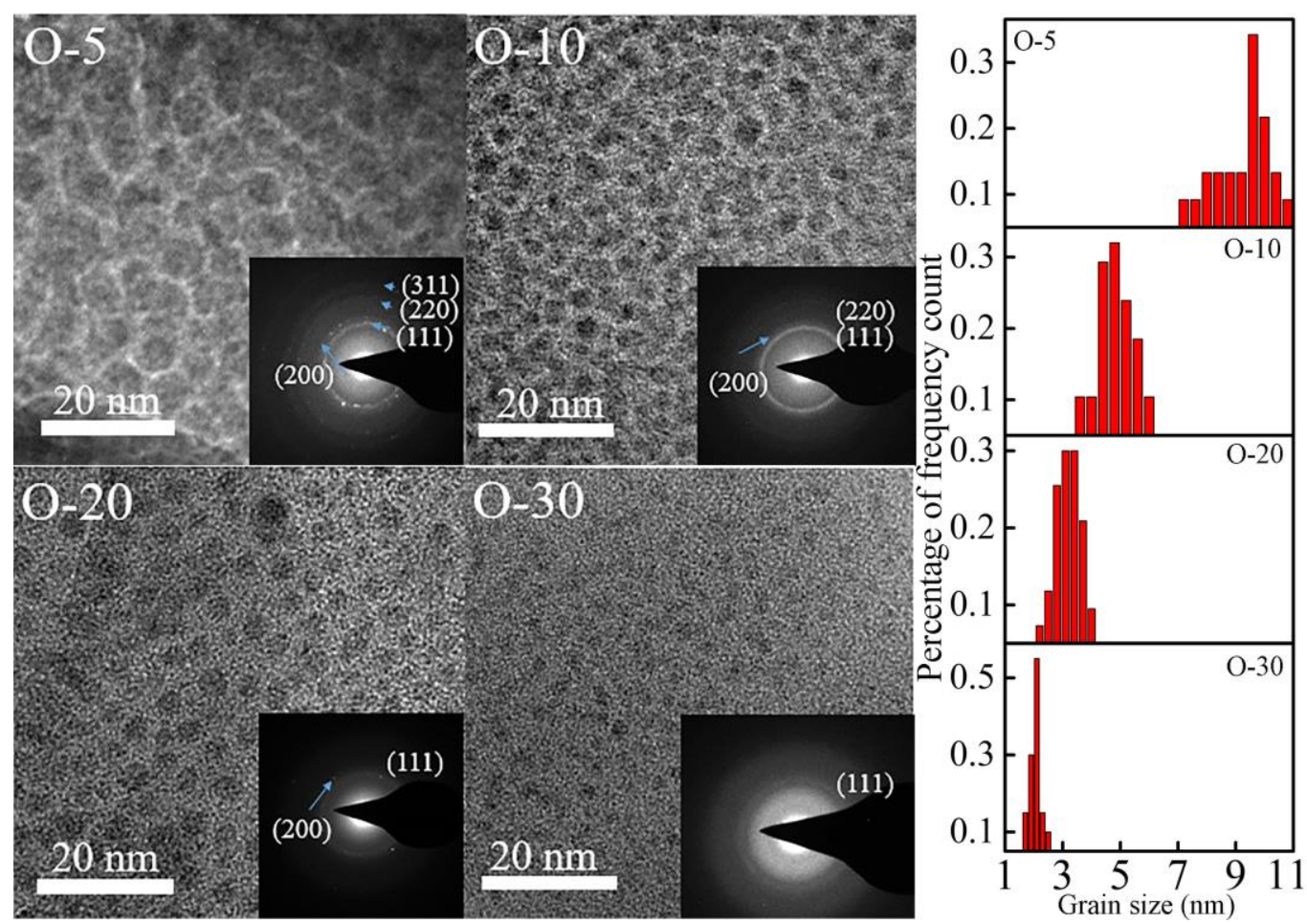

Figure 3. (Left image) Bright-field transmission electron microscopy (TEM) images of the O-5, O-10, O-20 and O-30 samples. The insets show the corresponding selected area electron diffraction (SAED) patterns. The diamond diffraction rings of the (111), (200), (220) and (311) planes are labelled in the SAED patterns. (Right) Particle size distribution of the samples O-5, O-10, O-20 and O-30.

The insets of Figure 3 show the evolution of the selected area electron diffraction (SAED) patterns of the samples. The SAED pattern of the O-5 sample contains sharp diffraction rings corresponding to the (111), (220), and (311) lattice planes of the diamond structure, confirming that the main content of the film is diamond. There is also a ring corresponding to the (200) plane of n-diamond (n-D), a metastable form of diamond with space group Fm3m symmetry and a cell parameter of $0.356 \mathrm{~nm}$ [29-31]. After oxygen plasma treatment for $10 \mathrm{~min}$, the SAED pattern contains diffraction rings corresponding to the (111), (220), and (200) planes, while the (311) plane 
diffraction ring disappears. With a treatment duration of $20 \mathrm{~min}$, SAED pattern exhibits (111) plane, and the (220) and (311) diffraction rings both disappear. It is also difficult to observe the (200) diffraction ring. After 30 min of oxygen plasma treatment, there is only a (111) diffraction ring, and the (200) ring also disappears. A larger Miller index corresponds to a larger unit cell. Thus, the disappearance of the (311) and (220) diffraction rings can be attributed to a decrease in the diamond grain size. However, as the (200) diffraction ring is very close to the (111) diffraction ring, its disappearance can be ascribed to the decrease in grain size or the diminishment of the $\mathrm{n}$-D phase after $30 \mathrm{~min}$ of oxygen plasma treatment. Above results discover the crystal plane evolution of nanocrystalline diamond grains with oxygen plasma treatment duration.

Furthermore, Figure 4 shows the magnified HRTEM images of the granular structure. The insets, (1), (2) and (3), show the Fourier transform (FT) diffractograms of the selected areas outlined by red squares, indicating the composition evolution of the films. The phase of the areas outlined by red squares is labelled in the bottom left of the FT images (cubic diamond (c-D), n-diamond (n-D), graphite (G) and amorphous carbon (a-C)). And FT image (0) corresponds to entire structure image. In the O-5 sample, areas (1) and (2) are c-D, $10 \mathrm{~nm}$ in size, with a (111) diffraction spot shown in the corresponding FT image, and area (3), the grain boundary zone of the O-5 sample, is a-C with a prominent diffused ring exists in the centre of the FT image. In FT image (0) of the O-5 sample, (200) diffraction spot can be observed indicating the presence of n-D. In the O-10 sample, c-D and n-D structures, $\sim 5 \mathrm{~nm}$ in size, can 


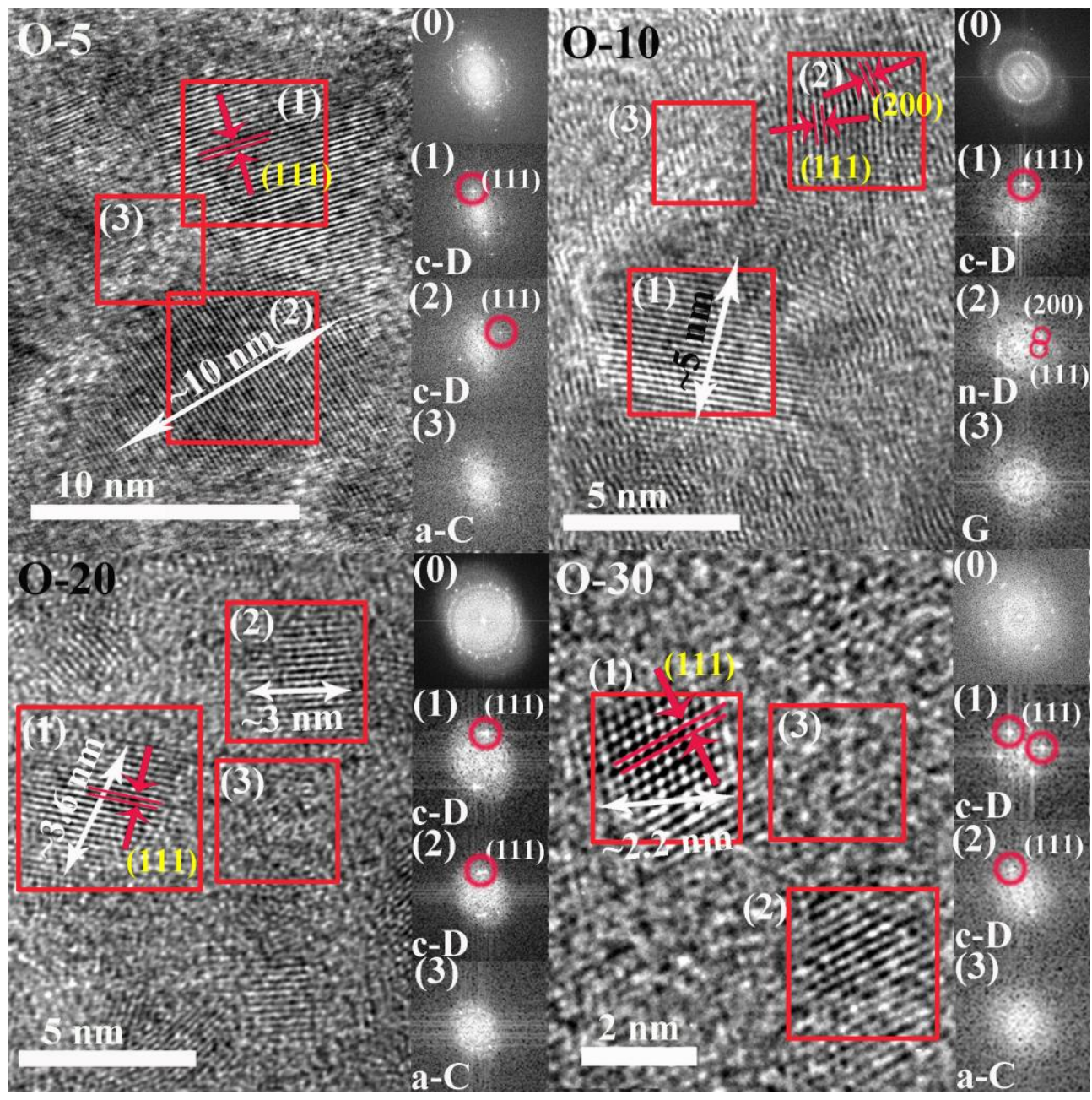

Figure 4. High-resolution transmission electron microscopy (HRTEM) images of the O-5, O-10, O-20 and O-30 samples. The insets on the right side show the Fourier transform (FT) images corresponding to the areas outlined by red squares. In addition, the phase of the areas outlined by red squares is labelled in the bottom left of the FT images (cubic diamond (c-D), n-diamond (n-D), graphite (G) and amorphous carbon (a-C)). Inset FT image (0) corresponds to entire structure image.

also be found in areas (1) and (2), respectively. The grain boundary zone of the O-10 sample contained graphite $(\mathrm{G})$ with a donut-shaped diffusion ring. In the O-20 sample, both c-D and n-D structures, $\sim 3 \mathrm{~nm}$ in size, are found. It is not easy to find the area in 
which c-D is close to n-D. Thus, here, to clearly show the diamond grain, only c-D is shown in the image. The (200) diffraction spot still can be observed in FT image (0) of O-20 sample. However, for the O-30 sample, it is easy to find the c-D structure, $\sim 2.2 \mathrm{~nm}$ in size, but the $\mathrm{n}-\mathrm{D}$ structure disappears. This reveals that metastable diamond diminishes. Nevertheless, a prominent diffused ring exists both in the centre of the SAED image of the O-30 sample and the FT image (3) of O-30 sample, indicating the presence of some sp2 phase or a-C. These suggest that $\mathrm{n}-\mathrm{D}$ (metastable diamond) is mainly transformed into a-C, which gives contribution to the disappearance of SiV PL.

To evaluate the photon statistics of $\mathrm{SiV}$ fluorescence in the diamond grains, we separated the particles from the O-20 sample using ultrasonic vibration. Then, confocal fluorescence scans were performed on the particles, as shown in Figure 5. Figure 5 (a) demonstrates that the saturation intensity ranges from $1.6 \times 10^{4}$ to $3.8 \times 10^{4}$ counts per second per pixel, with lower counts on the edge of the particle. Thus, we measured an autocorrelation function $\mathrm{g}^{(2)}$ on the edge of the particle labelled by the red cross in Figure 5 (a). Fitting the background-corrected autocorrelation function and assuming emission from multiple identical emitters, we obtained $g^{(2)}(0)=0.64$, indicating the presence of three emitters within a fluorescent spot, as shown in Figure 5 (b) [32]. The corresponding PL spectrum in Figure 5 (d) displays a luminescent peak at $738 \mathrm{~nm}$ with a full width at half maximum (FWHM) of approximately $6.4 \mathrm{~nm}$, confirming that the emitters are $\mathrm{SiV}$ centres. This indicates that $\mathrm{SiV}$ fluorescence from three emitters has been found on the edge of the particle. 

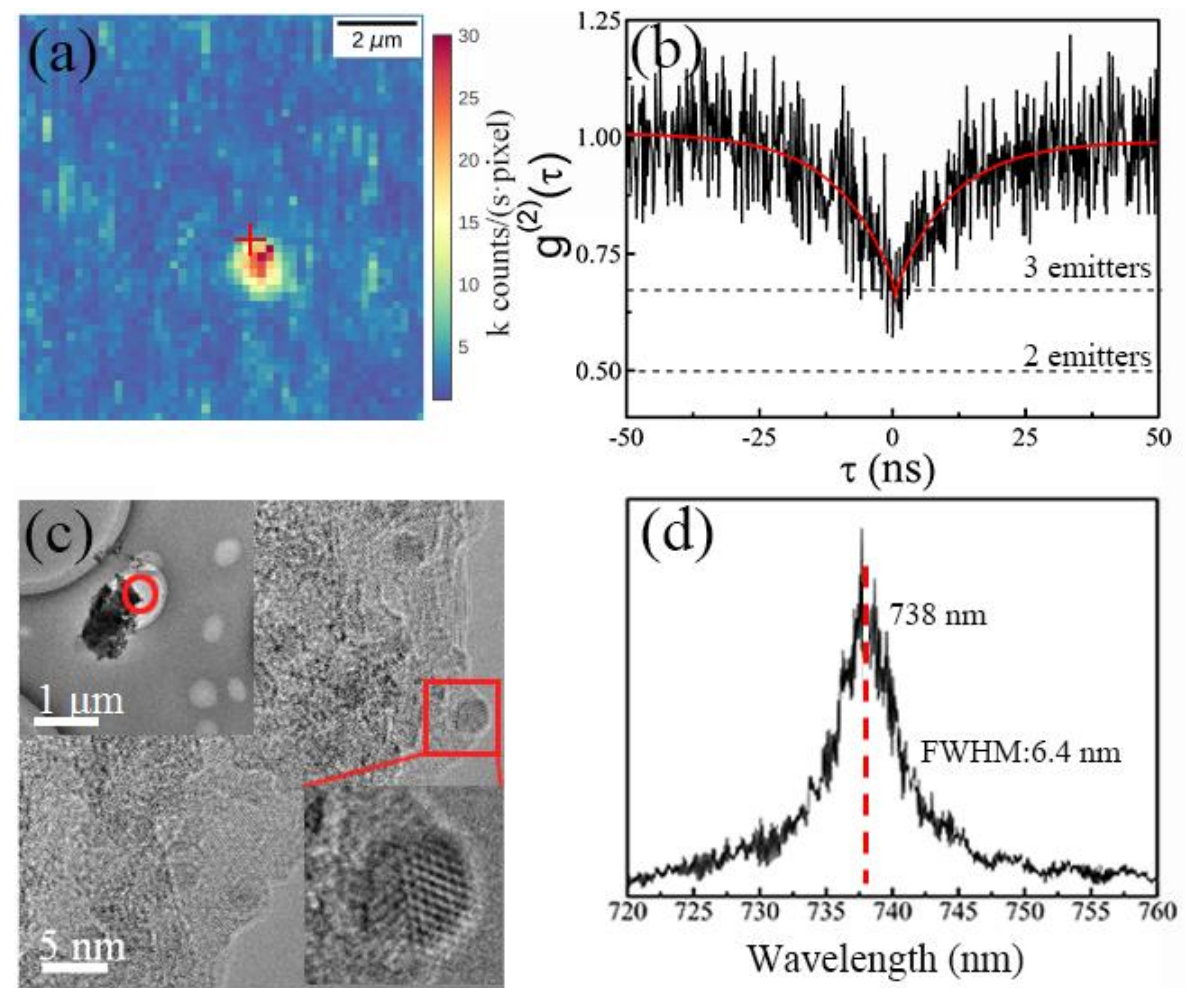

Figure 5. Detailed analysis of a diamond grain with a sub-4-nm size, containing less than three silicon-vacancy (SiV) emitters. (a) Confocal fluorescence image obtained using a fluorescent bandpass filter around $\mathrm{SiV}$ fluorescence, in which the excitation laser wavelength was $532 \mathrm{~nm}$. Pixel size was 200*200 $\mathrm{nm}^{2}$. (b) Autocorrelation function showing the background-corrected raw data (black line) and its fitted line (red). (c) High-resolution transmission electron microscopy (HRTEM) image of a particle obtained from the $\mathrm{O}-20$ sample showing a few isolated diamond grains on the edge of the particle. Left inset: TEM image of the particle showing the full view. The red circle denotes the area at which the HRTEM image was obtained. Right inset: enlarged image of the red square in the HRTEM image. (d) Background-subtracted $\mathrm{SiV}$ photoluminescence (PL) spectra of the particle in (a) measured at room temperature with 532-nm laser excitation. 
Furthermore, we used HRTEM to observe the microstructure of the particle, as shown in Figure 5 (c). The isolated nanocrystalline diamond grains with a size of approximately $2.5 \mathrm{~nm}$ are distributed at the edge of the particle, and their shape is displayed in the inset of Figure 5 (c). As the fluorescent spots of the confocal fluorescence scans are on the micron scale, there are tens of sub-4-nm diamond grains in the fluorescent spot. This indicates that fewer than three emitters are present in one nanocrystalline diamond grain. All these data confirm that we have prepared sub-4-nm diamond grains that can emit SiV PL with less than three emitters. Assuming that the emitting centers have the same probability existing in each grain, it would be of high probability for the emitters distribute in different grains, i.e., one diamond grain only contains one $\mathrm{SiV}$ center. It suggests that we can obtain nanodiamond grains capable of emitting single photon by reducing the grain size to the minimum. These results have great significance for nanodiamond-based applications involving single-photon sources, nanophotonics platform and quantum communications.

\section{Conclusions}

In summary, we reduced the size of diamond grains by treating nanocrystalline diamond films with oxygen plasma. Ultrafine diamond grains in size of 2.1-4 nm (sub-4-nm) that can emit SiV PL with less than three emitters were produced after 20 min of oxygen plasma etching. This approach so far provides the smallest man-made photoluminescent nanodiamonds with less than three $\mathrm{SiV}$ emitters, which have significant potential for applications involving single-photon sources, nanophotonics 
platform and quantum communications. Our results open a way to controllably adjust the size and properties of nanomaterials.

\section{Experimental Section}

4.1 Preparation of diamond film: The nanocrystalline diamond films were deposited on mirror-polished Si wafers in a MPECVD system. A mixture of $\mathrm{CH}_{4}$ and $\mathrm{Ar}$, at a flow rate of 4 and $196 \mathrm{sccm}$, respectively, was used as the reactant gas for the microwave discharge. The plasma was induced with a microwave power of $1200 \mathrm{~W}$ and a pressure of 150 Torr. All samples were grown for $60 \mathrm{~min}$, and no external heater was used to heat the substrate. The substrate was heated by plasma bombardment to a temperature of $470{ }^{\circ} \mathrm{C}$, which was monitored by a thermocouple embedded in the stainless-steel substrate holder.

4.2 Plasma bombardment: A mixture of $\mathrm{O}_{2}$ and $\mathrm{N}_{2}$, at flow rates of 20 and $80 \mathrm{sccm}$, respectively, was used as the reactant gas for the oxygen plasma. The plasma was induced with a microwave power of $900 \mathrm{~W}$ and a pressure of 0.5 Torr. Nanocrystalline diamond films were subjected to oxygen plasma bombardment for 5, 10,20 and $30 \min [33]$.

4.3 HBT measurement: Diamond particles were prepared for confocal imaging via common ultrasonication in alcohol. Ultrasonication was performed on the diamond film with the substrate for 4 hours. Alcohol containing the diamond particles was dropped onto a fused silica slide and rapidly dried. HBT measurements were performed on the nanodiamond grain using a home-made confocal setup. The resolution of our confocal microscope is $200 \mathrm{~nm}$. 
4.4 Other measurements: The luminescent properties of the samples were characterized by PL spectroscopy at a wavelength of $\lambda=532 \mathrm{~nm}$. The films were also analysed by visible Raman spectroscopy (EnSpectr R532) at a wavelength of $\lambda=532$ nm. The SiV luminescence was filtered out by a bandpass filter. The atomic concentrations of the present elements and their chemical bonds were characterized using XPS (Axis Ultra DLD spectrometer). The detailed microstructures were examined using TEM (Tecnai G2 F30 S-Twin produced by Philips-FEI).

\section{Author Contributions}

Chengke Chen and Xiaojun $\mathrm{Hu}$ designed the experiments. Chengke Chen and Yingshuang Mei performed the experiments and data analysis. Xiaojun $\mathrm{Hu}$, Xiao Li, Meiyan Jiang and Shaohua $\mathrm{Lu}$ commented on the data analysis and wrote the manuscript. Jingming Cui set up and performed the fluorescence measurements. All authors discussed the results and commented on the manuscript and have given approval to the final version of the manuscript.

\section{ACKNOWLEDGMENT}

This work was supported by the National Natural Science Foundation of China (Grant Nos. 50972129, 50602039, and 11504325) and the Natural Science Foundation of Zhejiang Province (LQ15A040004). This work was also supported by the International Science Technology Cooperation Program of China (2014DFR51160) and the National Key Research and Development Program of China (No. 2016YFE0133200) and European Union's Horizon 2020 Research and Innovation 
Staff Exchange (RISE) Scheme (No. 734578). This work was supported by the One Belt and One Road International Cooperation Project from Key Research and Development Program of Zhejiang Province (2018C04021). The authors wish to thank Prof. I-Nan Lin and his group for support with the MPECVD equipment.

\section{REFERENCES}

[1] Aharonovich I, Castelletto S, Simpson DA, Su CH, Greentree AD, Prawer S. Diamond-based single-photon emitters. Reports on Progress in Physics. 2011;74(7):076501.

[2] Duan X, Ao Z, Zhang H, Saunders M, Sun H, Shao Z, et al. Nanodiamonds in $\mathrm{sp}^{2} / \mathrm{sp}^{3}$ configuration for radical to nonradical oxidation: Core-shell layer dependence. Applied Catalysis B: Environmental. 2018;222:176-81.

[3] Duan X, Ao Z, Li D, Sun H, Zhou L, Suvorova A, et al. Surface-tailored nanodiamonds as excellent metal-free catalysts for organic oxidation. Carbon. 2016;103:404-11.

[4] Say JM, van Vreden C, Reilly DJ, Brown LJ, Rabeau JR, King NJC. Luminescent nanodiamonds for biomedical applications. Biophysical Reviews. 2011;3(4):171-84.

[5] He X-F, Manson NB, Fisk PT. Paramagnetic resonance of photoexcited N-V defects in diamond. II. Hyperfine interaction with the N 14 nucleus. Physical Review B. 1993;47(14):8816.

[6] He X-F, Manson NB, Fisk PT. Paramagnetic resonance of photoexcited N-V defects in diamond. I. Level anticrossing in the 3 A ground state. Physical Review B. 1993;47(14):8809. 
[7] Fyta M. Nitrogen-Vacancy Centers and Dopants in Ultrathin Diamond Films: Electronic Structure. The Journal of Physical Chemistry C. 2013;117(41):21376-81.

[8] Bradac C, Gaebel T, Naidoo N, Sellars MJ, Twamley J, Brown LJ, et al. Observation and control of blinking nitrogen-vacancy centres in discrete nanodiamonds. Nature nanotechnology. 2010;5(5):345-9.

[9] Vlasov, II, Shenderova O, Turner S, Lebedev OI, Basov AA, Sildos I, et al. Nitrogen and luminescent nitrogen-vacancy defects in detonation nanodiamond. Small. 2010;6(5):687-94.

[10] Nelz R, Fuchs P, Opaluch O, Sonusen S, Savenko N, Podgursky V, et al. Color center fluorescence and spin manipulation in single crystal, pyramidal diamond tips. Applied Physics Letters. 2016;109(19):193105.

[11] Chen Y-C, Salter PS, Knauer S, Weng L, Frangeskou AC, Stephen CJ, et al. Laser writing of coherent colour centres in diamond. Nature Photonics. 2016;11(2):77-80.

[12] Neumann P, Kolesov R, Jacques V, Beck J, Tisler J, Batalov A, et al. Excited-state spectroscopy of single NV defects in diamond using optically detected magnetic resonance. New Journal of Physics. 2009;11(1):013017.

[13] Tisler J, Balasubramanian G, Naydenov B, Kolesov R, Grotz B, Reuter R, et al. Fluorescence and spin properties of defects in single digit nanodiamonds. ACS nano. 2009;3(7):1959-65. 
[14] Lim T-S, Fu C-C, Lee K-C, Lee H-Y, Chen K, Cheng W-F, et al. Fluorescence enhancement and lifetime modification of single nanodiamonds near a nanocrystalline silver surface. Physical Chemistry Chemical Physics. 2009;11(10):1508-14.

[15] Li K, Zhou Y, Rasmita A, Aharonovich I, Gao WB. Nonblinking Emitters with Nearly Lifetime-Limited Linewidths in CVD Nanodiamonds. Physical Review Applied. 2016;6(2).

[16] Evans RE, Sipahigil A, Sukachev DD, Zibrov AS, Lukin MD. Narrow-Linewidth Homogeneous Optical Emitters in Diamond Nanostructures via Silicon Ion Implantation. Physical Review Applied. 2016;5(4).

[17] Vlasov, II, Shiryaev AA, Rendler T, Steinert S, Lee SY, Antonov D, et al. Molecular-sized fluorescent nanodiamonds. Nature nanotechnology. 2014;9(1):54-8.

[18] Vlasov II, Barnard AS, Ralchenko VG, Lebedev OI, Kanzyuba MV, Saveliev AV, et al. Nanodiamond Photoemitters Based on Strong Narrow-Band Luminescence from Silicon-Vacancy Defects. Advanced materials. 2009;21(7):808-12.

[19] Grudinkin SA, Feoktistov NA, Baranov MA, Smirnov AN, Davydov VY, Golubev VG. Low-strain heteroepitaxial nanodiamonds: fabrication and photoluminescence of silicon-vacancy colour centres. Nanotechnology. 2016;27(39):395606.

[20] Michaelson S, Hoffman A. Hydrogen bonding, content and thermal stability in nano-diamond films. Diamond and Related Materials. 2006;15(4-8):486-97.

[21] Ferrari AC, Robertson J. Origin of the $1150 \mathrm{~cm}-1$ Raman mode in nanocrystalline diamond. Physical Review B. 2001;63(12):121405. 
[22] Kuzmany H, Pfeiffer R, Salk N, Günther B. The mystery of the $1140 \mathrm{~cm}-1$ Raman line in nanocrystalline diamond films. Carbon. 2004;42(5-6):911-7.

[23] Gilkes KWR, Sands HS, Batchelder DN, Robertson J, Milne WI. Direct observation of sp3 bonding in tetrahedral amorphous carbon using ultraviolet Raman spectroscopy. Applied Physics Letters. 1997;70(15):1980-2.

[24] Sankaran KJ, Panda K, Sundaravel B, Tai NH, Lin IN. Enhancing electrical conductivity and electron field emission properties of ultrananocrystalline diamond films by copper ion implantation and annealing. Journal of Applied Physics. 2014;115(6):063701.

[25] Ahmed MH, Byrne JA, McLaughlin JAD, Elhissi A, Ahmed W. Comparison between FTIR and XPS characterization of amino acid glycine adsorption onto diamond-like carbon (DLC) and silicon doped DLC. Applied Surface Science. 2013;273:507-14.

[26] Mei Y, Fan D, Lu S, Shen Y, Hu X. SiV center photoluminescence induced by $\mathrm{C}=\mathrm{O}$ termination in nanocrystalline diamond and graphite loops hybridized films. Journal of Applied Physics. 2016;120(22):225107.

[27] Sternberg M, Zapol P, Curtiss L. Carbon dimers on the diamond (100) surface:Growth and nucleation. Physical Review B. 2003;68(20).

[28] Zapol P, Sternberg M, Curtiss LA, Frauenheim T, Gruen DM. Tight-binding molecular-dynamics simulation of impurities in ultrananocrystalline diamond grain boundaries. Physical Review B. 2001;65(4):045403. 
[29] Kovarik P, Bourdon E, Prince R. Electron-energy-loss characterization of laser-deposited a-C, a-C: H, and diamond films. Physical Review B. 1993;48(16):12123.

[30] Prawer S, Peng J, Orwa J, McCallum J, Jamieson D, Bursill L. Size dependence of structural stability in nanocrystalline diamond. Physical Review B. 2000;62(24):R16360.

[31] Hirai H, Kondo K-i. Modified phases of diamond formed under shock compression and rapid quenching. Science. 1991;253(5021):772.

[32] Wang C, Kurtsiefer C, Weinfurter H, Burchard B. Single photon emission from $\mathrm{SiV}$ centres in diamond produced by ion implantation. Journal of Physics B: Atomic, Molecular and Optical Physics. 2005;39(1):37.

[33] $\mathrm{Hu} \mathrm{X}$, Chen C, inventors; Ultrafine sized nanodiamond grains with $\mathrm{Si}-\mathrm{V}$ luminescence and their preparation method. Chinese patent 201610870721.7, 2016, and PCT/CN2017/103313, 2017. 